\title{
Open
}

\section{Mutation of WIF1: a potential novel cause of a Nail-Patella-like disorder}

\author{
Marilyn C. Jones, MD'1, Sarah E. Topol, RN², Manuel Rueda, PhD², Glenn Oliveira, BS², \\ Tierney Phillips, BA², Emily G. Spencer, PhD² and Ali Torkamani, PhD²,3
}

\begin{abstract}
Purpose: Nail-Patella syndrome is a dominantly inherited genetic disorder characterized by abnormalities of the nails, knees, elbows, and pelvis. Nail abnormalities are the most constant feature of NailPatella syndrome. Pathogenic mutations in a single gene, $L M X 1 B$, a mesenchymal determinant of dorsal-ventral patterning, explain approximately $95 \%$ of Nail-Patella syndrome cases. However, $5 \%$ of cases remain unexplained.
\end{abstract}

Methods: Here, we present exome sequencing and analysis of four generations of a family with a dominantly inherited Nail-Patella-like disorder (nail dysplasia with some features of Nail-Patella syndrome) who tested negative for $L M X 1 B$ mutation.
Results: We identify a loss-of-function mutation in WIF1 (NM_007191 p.W15*), which is involved in mesoderm segmentation, as the suspected cause of the Nail-Patella-like disorder observed in this family.

Conclusions: Mutation of WIF1 is a potential novel cause of a NailPatella-like disorder. Testing of additional patients negative for $L M X 1 B$ mutation is needed to confirm this finding and further clarify the phenotype.

Genet Med advance online publication 6 April 2017

Key Words: Fong disease; HOOD syndrome; nail dysplasia; Nail-Patella syndrome; Turner-Kieser syndrome
Nail-Patella syndrome is an autosomal dominant multisystem disorder primarily affecting skeletal and connective tissues. Primary features of the syndrome include dysplasia of the nails, knees, and elbows. Nails are typically hypoplastic, absent, or dystrophic. Patellae are often small or irregularly shaped. Elbow abnormalities include dysplasia of the radial head and hypoplasia of the lateral epicondyle and capitellum. Joints may swell, dislocate, display hypermobility, or display early degenerative arthritis. Iliac horns are observed radiologically in approximately $70 \%$ of cases. Other features include renal dysfunction and, less frequently, glaucoma. Renal dysfunction is observed in approximately $40 \%$ of cases, with end-stage renal diseases occurring in approximately $5 \%$ of cases. Glaucoma is observed more frequently and at a younger age in this population. The overall presentation can vary widely from one affected individual to another, with various manifestations ranging from severe to totally absent on a case-by-case basis. Nail changes are the most constant feature of Nail-Patella syndrome and are observed in approximately $98 \%$ of cases. The incidence of NailPatella syndrome is thought to be 1 in 50,000 ${ }^{1,2}$

Loss-of-function mutations in $L M X 1 B$ are the only known genetic cause of Nail-Patella syndrome. $L M X 1 B$ is a WNT signaling-responsive transcription factor crucial for mesenchymal dorsoventral patterning in developing limbs. ${ }^{3} \mathrm{WNT}$ signaling plays a major role in a wide variety of embryonic development processes, and it is thought that the timing and restricted expression of $L M X 1 B$, as well as other cooperating and compensating signaling pathways, underlie both the constraints on abnormalities typically observed in Nail-Patella syndrome and the heterogeneity of clinical presentation observed from patient to patient. ${ }^{4}$ LMX1B mutations account for $95 \%$ of all Nail-Patella syndrome cases, ${ }^{2}$ leaving some cases unexplained even after comprehensive $L M X 1 B$ mutation profiling.

Here, we present the exome sequencing findings for four generations of a family with nail dysplasia and some features of Nail-Patella syndrome. Prior to referral for exome sequencing, the proband of this family underwent workup for NailPatella syndrome; however, her pelvic radiograph results were negative for iliac horns, her patellas were not small, and test results were negative for $L M X 1 B$ mutation. Although many of the classic findings for Nail-Patella syndrome were not observed, given the similarity of the nail dysplasia observed in this family to that observed in Nail-Patella syndrome and the co-occurrence of joint laxity, the case was referred for exome sequencing for a suspected Nail-Patella-like disorder or other connective-tissue disorder. Exome sequencing, bioinformatic analysis, and application of standard inheritancebased, population-based, and coding impact filters resulted in the conclusion that a loss-of-function mutation in WIF1, a WNT signaling regulator involved in mesoderm segmentation, ${ }^{5}$ was the suspected novel cause of the Nail-Patella-like disorder observed in this family. 


\section{MATERIALS AND METHODS}

\section{Study consent}

Study participants provided written informed consent and the protocol was approved by the institutional review board of Scripps.

\section{Whole-exome sequencing, variant calling, and filtration}

DNA was extracted from freshly drawn blood and wholeexome sequencing (WES) was pursued utilizing Agilent SureSelect exome hybridization followed by barcoding and sequencing of paired 100-bp reads on an Illumina HiSeq2500 instrument. Read mapping, variant calling, and quality filtration were performed using a BWA-GATK best-practices variant quality score recalibration approach. Mean coverage of $86 \times$ per individual was achieved with $98.1 \%$ of the target exome covered by $>10$ reads. Variant annotation was performed using the SG-ADVISER system. ${ }^{4}$ A series of filters was applied to derive a set of candidate disease-causative variants (Table 2): (i) population-based filtration, because of the rarity of this condition, removed variants present at $>0.2 \%$ allele frequency in the Exome Aggregation Consortium, ${ }^{6} 1000$ Genomes, ${ }^{7}$ the NHLBI Exome Sequencing Project, ${ }^{8}$ or Scripps Wellderly populations; ${ }^{9}$ (ii) functional impact-based filtration to remove variants that are not nonsynonymous, frameshift, in-frame, nonsense, or do not affect canonical splice-site donor/acceptor sites; and (iii) inheritance-based filters to remove variants that do not segregate in the family in a manner consistent with autosomal dominant inheritance.

\section{RESULTS}

\section{Phenotype}

The proband was a 45-year-old woman presenting with dysplastic ridged nails, and joint laxity. She had a history of multiple fractures with minimal trauma; a torn anterior cruciate ligament; subluxations of the left shoulder, knee, and temporomandibular joints; spondylolisthesis; and arthritis. Prior to referral for exome sequencing, she was evaluated for Nail-Patella syndrome with radiographs of the knees and pelvis, which documented patellas of normal size and no iliac horns. Genetic testing for pathogenic deletions, duplications, and mutations in $L M X 1 B$ was negative (Fulgent Diagnostics).

Nail dysplasia and joint laxity co-occur in a variety of disorders, all of which are associated with additional severe findings. A search for disorders with combined nail dysplasia (human phenotype ontology identifier HP:0002164) and joint laxity (HP:0001388) via the Phenomizer tool ${ }^{10}$ returned possible diagnoses that were severe syndromic disorders (Meier-Gorlin syndrome, Costello syndrome, chromosome 2q32-q33 deletion syndrome, Weaver syndrome, Coffin-Lowry syndrome, craniofrontonasal syndrome, and focal dermal hypoplasia), severe bone development disorders (metaphyseal chondrodysplasia and cranioectodermal dysplasia), or Nail-Patella syndrome. Therefore, although many of the classic findings for Nail-Patella syndrome were not observed, we concluded that the phenotype observed in the proband was nail dysplasia with some features of Nail-Patella syndrome, or what we refer to as a Nail-Patella-like disorder.

Family history included four generations of 14 total individuals with nail dysplasia and a variety of connective-tissue concerns (Table 1, Figure 1, Supplementary Figures S2 and S3 online). All affected family members belonged to the maternal lineage of the proband, including the mother, grandmother, and maternal aunts, uncles, cousins, brother, and niece of the proband (see pedigree in Figure 2); there were two instances of male-to-male transmission supporting autosomal dominant inheritance in the maternal lineage. To identify candidate genetic variants underlying the Nail-Patella-like disorder observed in four generations of this family, WES was pursued for five individuals in the family, the mother-father-proband trio and two cousins (the most distantly related family members available for sequencing). Follow-up genotyping of candidate variants was performed in these individuals plus an additional five members of the family.

Table 1 Phenotype description

\begin{tabular}{|c|c|c|c|c|c|c|c|}
\hline Subject & Age & Nail dysplasia & Iliac horns & Joint problems & Renal disease & Glaucoma & Other \\
\hline ID41P (proband) & 45 & Present & Absent & $\begin{array}{l}\text { Lax joints, multiple subluxation, torn } \\
\text { ACL, multiple fractures, arthritis }\end{array}$ & & & $\begin{array}{l}\text { Irritable bowel } \\
\text { syndrome }\end{array}$ \\
\hline ID41M (mother) & 73 & Present & Unknown & $\begin{array}{l}\text { Patellar tendon rupture, } 3 \text { knee } \\
\text { replacements, scoliosis }\end{array}$ & $\begin{array}{l}\text { Chronic kidney } \\
\text { disease }\end{array}$ & Present & $\begin{array}{l}\text { Breast cancer, } \\
\text { chronic cough }\end{array}$ \\
\hline ID41K4 (cousin) & 48 & Present & Unknown & & & & \\
\hline ID41K5 (cousin) & NA & Present & Unknown & & & & Crohn's disease \\
\hline ID41K6 (daughter) & 15 & Present & Absent & $\begin{array}{l}\text { Hip dysplasia, torn ulnar collateral } \\
\text { ligament, chronic back pain }\end{array}$ & & & \\
\hline ID41K7 (daughter) & 13 & Present & Unknown & $\begin{array}{l}\text { Recurrent ankle sprain, } \\
\text { microinstability at fibular fracture }\end{array}$ & & & \\
\hline ID41K8 (son) & 13 & Present & Unknown & Torn ankle tendon & & & $\begin{array}{l}\text { Cranial nerve } \\
\text { palsy }\end{array}$ \\
\hline ID41K9 (uncle) & NA & Present & Unknown & & & & Chronic cough \\
\hline ID41K10 (aunt) & NA & Present & Unknown & $\begin{array}{l}\text { Multiple joint symptoms, } \\
\text { hip replacement }\end{array}$ & & & Chronic cough \\
\hline
\end{tabular}

All familial relations are maternal in respect to the proband. 
a

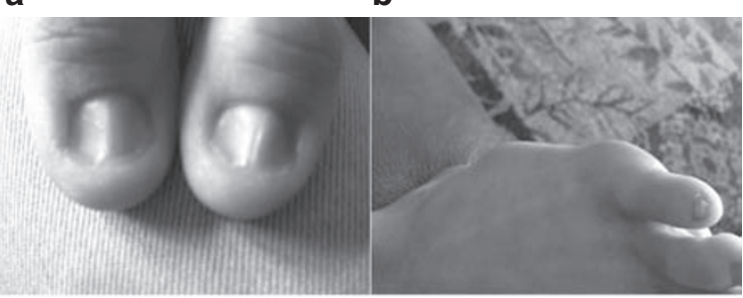

C

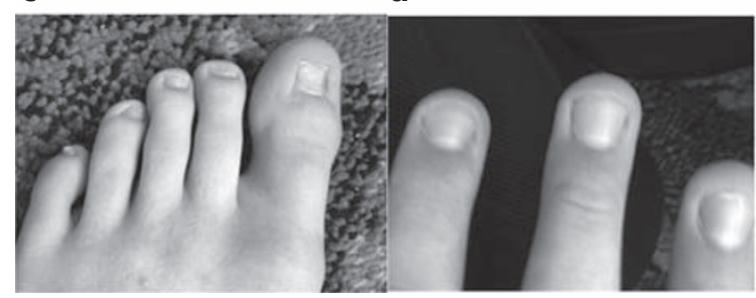

Figure 1 Images of the proband and children's nail dysplasia. (a) Both thumbs of the proband (ID41P). (b) Fifth toenail of the proband's daughter (ID41K7). (c,d) Toenails and fingernails of the proband's son (ID41K8). Note the variable ridging, dysplasia, and thin texture.

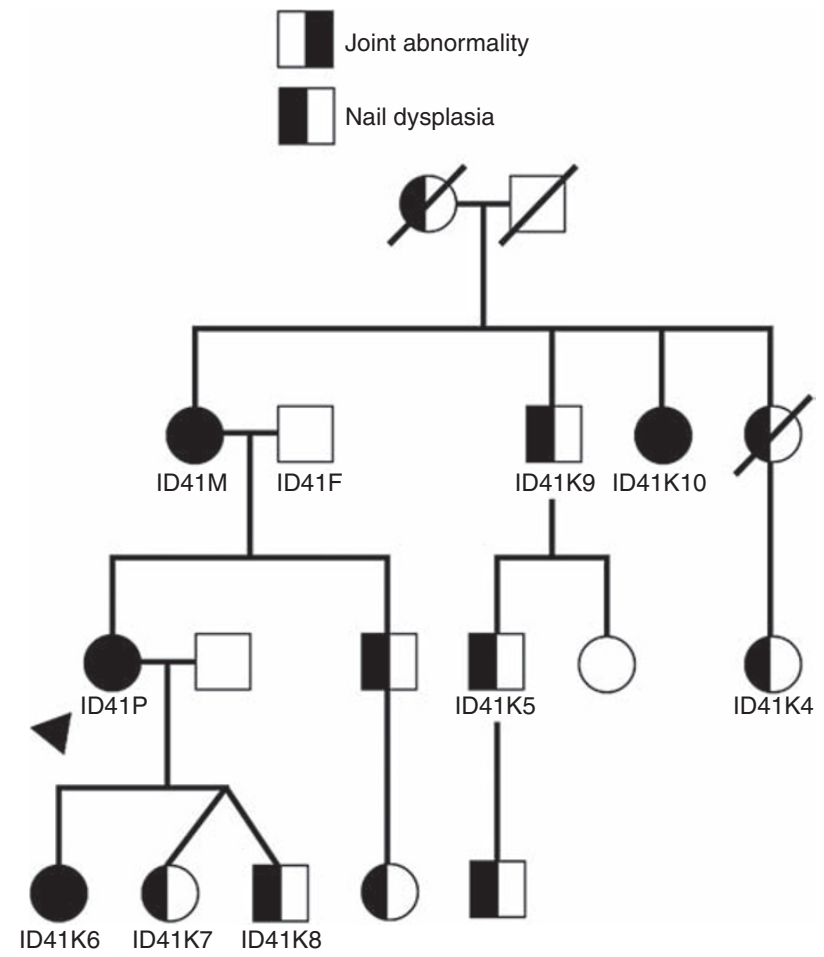

Figure 2 Family pedigree. Pedigree of four generations of a family with a dominantly inherited Nail-Patella-like disorder. The proband is indicated by the arrow. Identifiers are provided for family members that underwent genetic analysis. Corresponding identifiers can be found in the Tables. Not all unaffected family members are depicted. Unaffected family members not pictured include three unaffected children of the pictured brother of the proband, four unaffected children of ID41K10, and an unaffected brother of ID41K4.

\section{Sequencing and candidate variant identification}

WES ( $\sim 86 \times$ coverage, $98.1 \%$ of target exome covered by $10+$ reads) was performed for the affected proband (ID41P), unaffected father (ID41F), affected mother (ID41M), and two affected maternal cousins (ID41K4 and ID41K5) (see Materials and Methods for details) (Figure 1b). Variants discovered by WES were annotated by SG-ADVISER. ${ }^{4}$ Nonsynonymous, inframe, frameshift, nonsense, and consensus splice site variants, at a threshold population allele frequency of $<0.2 \%$, were filtered under an autosomal dominant model of inheritance. This allele frequency threshold is 100 -fold higher than the incidence of Nail-Patella syndrome (1 in 50,000). Twelve candidate causative variants were retained after filtration (Table 2).

Follow-up genotyping, via Sanger sequencing, for the 12 candidate variants was performed in the above mentioned individuals plus three affected children (ID41K6, ID41K7, ID41K8), an affected maternal uncle (ID41K9), and an affected maternal aunt (ID41K10) of the proband (Figure 1b). This process ultimately resulted in the retention of three candidate variants: KRT74 p.R379L, RDH16 p.R153G, and WIF1 p.W15* (shown in boldface in Table 2).

\section{DISCUSSION}

Relaxed allele frequency-based filtration of coding variants inherited in an autosomal dominant fashion in the study family resulted in three candidate genes for the observed Nail-Patellalike disorder: KRT74, RDH16, and WIF1. It should be noted that the underlying assumption leading to the identification of these three candidate variants was that the disorder is driven by a coding mutation captured by exome sequencing, and that the Nail-Patella-like disorder observed in this family is rare. No assumptions regarding the specific phenotype were included in this variant prioritization approach-i.e., variants underlying other known causes of nail dysplasia or other connective-tissue disorders would have been captured by our analysis approach. Given our initial assumptions, further filtration of the candidate variants by allele frequency under the assumption of complete penetrance, and prioritization based on biological relevance of the candidate genes, resulted in the conclusion that mutation of WIF1 was the suspected cause of Nail-Patella-like disorder in this family.

The candidate variant in KRT74 can probably be removed from consideration on the basis of phenotype as well as allele frequency. KRT74 is an epithelial keratin known to cause autosomal dominant woolly hair and autosomal recessive ectodermal dysplasia. ${ }^{11}$ The family in this report had normal hair, teeth, skin, and sweat glands. Moreover, this particular variant in KRT74 (rs143748352) has been observed at $0.047 \%$ allele frequency in Europeans, ${ }^{6}$ which is an approximately 25 -fold excess of the incidence of Nail-Patella syndrome. Therefore, under the assumption of near-complete penetrance, which was appropriate given the pattern of inheritance observed in this family, the variant in KRT74 was unlikely to be a cause of the observed Nail-Patella-like disorder. However, we could not fully exclude the possibility that the variant contributed to a portion of the phenotype observed in this family.

The candidate variant in $\mathrm{RDH} 16$ cannot definitively be eliminated from consideration due to allele frequency, but its known 


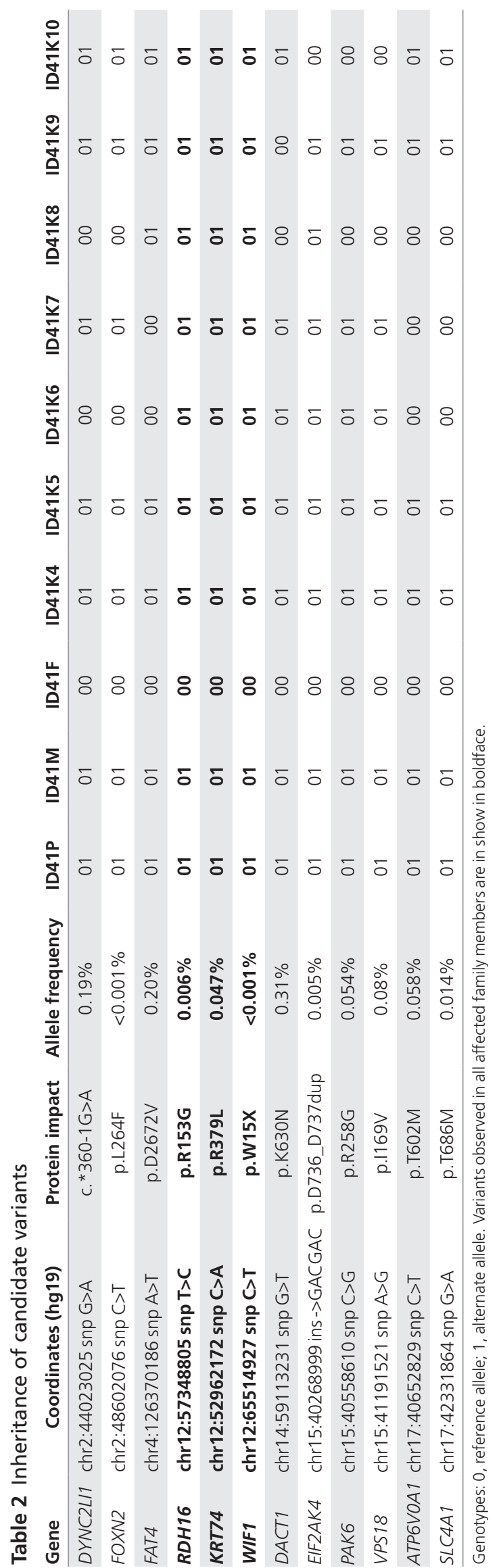

function does not appear to fit the observed phenotype. The variant in RDH16 (rs372580365) has been observed at $0.006 \%$ allele frequency in Europeans, ${ }^{6}$ which is slightly $(3 \times)$ greater than the incidence of Nail-Patella syndrome-an excess that would be amplified further given that $95 \%$ of cases are already explained by mutation in $L M X 1 B$ mutation $(60 \times$ greater). Moreover, $\mathrm{RDH16}$ is a liver-specific retinol dehydrogenase that plays no known role in connective-tissue biology. Finally, RDH16 knockout mice have no significant abnormalities. ${ }^{12}$

However, the variant in WIF1 is a strong candidate as the cause of a novel Nail-Patella-like disorder. The nonsense variant (WIF1 p.W15*) observed in this gene has not previously been observed in more than 100,000 genomes. ${ }^{6}$ Therefore, the frequency of this variant is consistent with a fully penetrant cause of the observed disorder. Moreover, WIF1 is a secreted antagonist of WNT signaling, expressed predominantly at the superficial layer of epiphyseal and articular cartilage, and it is known to promote chondrogenesis. ${ }^{13}$ In mouse models of arthritis, loss of WIF1 aggravates cartilage damage. ${ }^{14}$ The observed nonsense mutation in WIF1 is expected to result in the complete loss of the $\mathrm{N}$-terminal signal peptide of WIF1 (amino acids 1-28) and thus inhibit its secretion into the extracellular matrix, where it inhibits WNT factors. Therefore, loss of WIF1 function would be expected to lead to joint cartilage abnormalities. Moreover, WIF1 functions within the same molecular signaling network as $L M X 1 B$ - a WNT-responsive transcription factor. ${ }^{15}$

The WNT pathway controls a variety of embryonic development processes. The specificity of WNT signaling is achieved through a variety of WNT ligands, each of which is subject to highly organized temporal and spatially restricted patterns of expression to coordinate embryonic development. ${ }^{16} \mathrm{~A}$ complex, and not fully understood, interplay between WNT and other signaling cascades is known to mediate joint development. ${ }^{17}$ Similarly, WNT signaling is known to play a central role in nail development, ${ }^{18}$ although the precise molecular mechanisms have not been well described. It is thought that the role of $L M X 1 B$ as both a necessary and sufficient mediator of WNT signaling to specify dorsal limb patterning, specifically through induction by the WNT ligand WNT7A, underlies the primary abnormalities observed in Nail-Patella syndrome. ${ }^{15}$ However, $L M X 1 B$ is also involved in a variety of other complex WNT-dependent processes, in which $L M X 1 B$ function may be compensated for by other factors, potentially leading to the phenotypic heterogeneity observed across Nail-Patella syndrome cases. ${ }^{19}$ Similarly, WIF1 interacts with a variety of WNT ligands, including WNT7A, and thus participates in an overlapping set of WNT signaling functions as $L M X 1 B .{ }^{20}$ However, the details of the role of WIF1 in embryonic and other developmental processes have not been well described.

Further study of additional WIF1-positive cases would help elucidate the role of WIF1 mutation in Nail-Patella-like disorder and the role of WNT signaling in nail and joint development. The phenotype in this family evolves over time. The majority of affected individuals have abnormal nails at birth but do not manifest other features until they become competitively active 
during adolescence. Multiple joint dislocations and arthritis are most troublesome. Two older members of the family have developed renal issues and one has glaucoma. It is unclear whether these features are related to the WIF1 mutation or are unrelated. Longitudinal follow-up of family members is clearly warranted. The complete phenotypic spectrum due to loss of WIF1 function may be clarified if more families are reported.

\section{SUPPLEMENTARY MATERIAL}

Supplementary material is linked to the online version of the paper at http://www.nature.com/gim

\section{ACKNOWLEDGMENTS}

This work was supported by Scripps Genomic Medicine, an NIHNCATS Clinical and Translational Science Award (CTSA; 5 UL1 RR025774) to STSI, as well as funding from the Shaffer Family Foundation and the Anne and Henry Zarrow Foundation. Further support was received from the Lavin Family Foundation ( $\mathrm{NIH}-\mathrm{U} 01$ HG006476 and U54GM114833 to A.T.). We thank the following members of our review panel for their dedication and support: Kelly Bethel, Joel Diamant, Nelson Hywnn, James Mason, Evan Muse, Brad Patay, Paul Pockros, Ron Simon, and Gary Williams.

\section{DISCLOSURE}

The authors declare no conflict of interest.

\section{REFERENCES}

1. Sweeney E, Hoover-Fong JE, McIntosh I. Nail-Patella syndrome. In: Pagon RA, Adam MP, Ardinger HH, et al. (eds). GeneReviews. University of Washington: Seattle, WA, 13 November 2014

2. Sweeney E, Fryer A, Mountford R, Green A, McIntosh I. Nail patella syndrome: a review of the phenotype aided by developmental biology. I Med Genet 2003;40:153-162.

3. Chen $H$, Lun Y, Ovchinnikov D, et al. Limb and kidney defects in Lmx1b mutant mice suggest an involvement of LMX1B in human nail patella syndrome. Nat Genet 1998:19:51-55.

4. Pham PH, Shipman WJ, Erikson GA, Schork NJ, Torkamani A. Scripps genome ADVISER: annotation and distributed variant interpretation SERver. PLOS One 2015;10:e0116815.

5. Hsieh JC, Kodjabachian L, Rebbert ML, et al. A new secreted protein that binds to Wnt proteins and inhibits their activities. Nature 1999;398:431-436.

6. Lek M, Karczewski KJ, Minikel EV, et al.; Exome Aggregation Consortium. Analysis of protein-coding genetic variation in 60,706 humans. Nature 2016;536:285-291
7. Auton A, Brooks LD, Durbin RM, et al.; 1000 Genomes Project Consortium. A global reference for human genetic variation. Nature 2015;526:68-74.

8. Tennessen JA, Bigham AW, O'Connor TD, et al.; Broad GO; Seattle GO; NHLBI Exome Sequencing Project. Evolution and functional impact of rare coding variation from deep sequencing of human exomes. Science 2012;337:64-69.

9. Erikson GA, Bodian DL, Rueda M, et al. Whole-Genome Sequencing of a Healthy Aging Cohort. Cell 2016;165:1002-1011.

10. Köhler S, Schulz MH, Krawitz P, et al. Clinical diagnostics in human genetics with semantic similarity searches in ontologies. Am J Hum Genet 2009;85:457-464.

11. Raykova D, Klar J, Azhar A, et al. Autosomal recessive transmission of a rare KRT74 variant causes hair and nail ectodermal dysplasia: allelism with dominant woolly hair/hypotrichosis. PLoS One 2014;9:e93607.

12. van der Weyden L, White JK, Adams DJ, Logan DW. The mouse genetics toolkit: revealing function and mechanism. Genome Biol 2011;12:224.

13. Surmann-Schmitt $C$, Widmann N, Dietz $U$, et al. Wif-1 is expressed at cartilage-mesenchyme interfaces and impedes Wnt3a-mediated inhibition of chondrogenesis. J Cell Sci 2009;122(Pt 20):3627-3637.

14. Stock M, Böhm C, Scholtysek C, et al. Wht inhibitory factor 1 deficiency uncouples cartilage and bone destruction in tumor necrosis factor $\alpha$-mediated experimental arthritis. Arthritis Rheum 2013;65:2310-2322.

15. Chen $\mathrm{H}$, Johnson RL. Interactions between dorsal-ventral patterning genes $\operatorname{Im\times 1b}$, engrailed-1 and wnt-7a in the vertebrate limb. Int J Dev Biol 2002:46:937-941.

16. MacDonald BT, Tamai $\mathrm{K}$, He X. Wnt/beta-catenin signaling: components, mechanisms, and diseases. Dev Cell 2009;17:9-26.

17. Lories RJ, Corr M, Lane NE. To Wnt or not to Wnt: the bone and joint health dilemma. Nat Rev Rheumato/ 2013;9:328-339.

18. Naz G, Pasternack SM, Perrin C, et al. FZD6 encoding the Wnt receptor frizzled 6 is mutated in autosomal-recessive nail dysplasia. Br J Dermatol 2012;166:10881094.

19. Harita Y, Kitanaka S, Isojima T, Ashida A, Hattori M. Spectrum of LMX1B mutations: from nail-patella syndrome to isolated nephropathy. Pediatr Nephrol; e-pub ahead of print 23 July 2016.

20. Bányai L, Kerekes K, Patthy L. Characterization of a Wnt-binding site of the WIFdomain of Wnt inhibitory factor-1. FEBS Lett 2012;586:3122-3126.

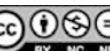

This work is licensed under a Creative Commons Attribution-NonCommercial-NoDerivs 4.0 International License. The images or other third party material in this article are included in the article's Creative Commons license, unless indicated otherwise in the credit line; if the material is not included under the Creative Commons license, users will need to obtain permission from the license holder to reproduce the material. To view a copy of this license, visit http://creativecommons.org/licenses/by-nc-nd/4.0/

(c) The Author(s) (2017) 